cone beam computed tomography and computer-assisted surgery, for example in hard tissue reconstruction in Chapter 13 , which can significantly reduce postoperative complications by simulating a surgical plan and fabricating a template to guide bone grafting.

I expect this will be a popular reference text for many oral and maxillofacial specialists. However, I feel it could benefit from further editing to break down the written content with more images and data reference boxes, thus appealing to a wider audience who have a particular interest in the topic.

\section{E. McCrory}

\section{THE ORTHODONTIC MINI-IMPLANT CLINICAL HANDBOOK}

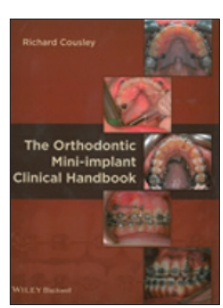

\section{R. Cousley}

Wiley-Blackwell

price $€ 49.99$; pp 184

ISBN 9781118275993

The orthodontic mini-implant clinical

handbook has been developed to guide professionals in a new and acceptable means of yielding anchorage within orthodontic treatment. It is strongly weighted towards maximising success within clinical practice. As the varied applications for these bone anchorage devices become more apparent, this book provides a foundation towards treatment in an evidence-based manner.

This lustrous hardback introduces the concepts, indications, contra-indications and biomechanics of orthodontic mini-implants. The early 11 chapters equip the reader with a sound understanding on which to build on. Richard Cousley provides a detailed approach in the pre-, peri and post-operative aspects of mini-implant insertion. Limitations and handy troubleshooting tips are emphasised throughout the text. This is particularly beneficial for clinicians who are actively adopting the technique into their routine practice. The majority of the handbook is dedicated to specific mini-implant applications, such as incisor retraction and asymmetry correction. Within these chapters, the author has included a clinical objective along with other alternative treatment options. This is valuable in enabling the audience to draw comparisons with conventional anchorage techniques. Each clinical scenario is supported with case examples and a literature reference, asserting the author's experience and knowledge in this field.

The handbook displays a host of colourful clinical photographs and illustrations. This brings the clinical information to life, and engages the reader with the concepts of this treatment modality. It also helps visualise the biomechanics involved, which are explained simply but effectively. The style of writing is easy to follow, with salient points highlighted in purple text boxes throughout each chapter. The layout utilises bullet points and subheadings to depict a step by step approach to mini-implants, a style described by the author as a 'cook-book', emphasising the very logical, concise nature of the book, which could easily be used as a reference tool. The orthodontic mini-implant clinical handbook is a compact, informative read for all orthodontic clinicians and personnel involved with treatment using mini-implants. I have no hesitation in recommending this book as an essential read for orthodontic specialty trainees who may benefit from the progressive approach.

C. Martin

\section{ADULT ORTHODONTICS}

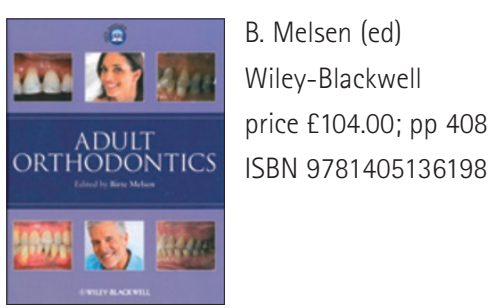

Adult orthodontics is not new, with the first case being recorded in 1901, however, since then patient age has increased significantly and the number of adults receiving treatment has escalated worldwide. This book has been superbly constructed by Birte Melsen, in conjunction with a host of other outstanding contributors, many of whom are considered leaders in this field. It seeks to demonstrate an unrestricted range of problems and solutions for adult patients, highlighting the skills and abilities required by clinicians to perform them.

The author establishes from the outset that the patient's presenting complaint is often only the 'tip of the iceberg' and for many adult patients managing expectations and achieving realistic results are key. Over its 20 chapters, the potential adult patient population groups are introduced, with their presenting complaints, orthodontic problems and treatment outcome. This is usually delivered by a series of clear clinical photographs, with accompanying, easily readable paragraphs. Additionally a companion website and CD-ROM provide supplemental case examples.

From her experience as a biologic researcher, Melsen gives us detailed background knowledge into the aetiology of malocclusions by means of vivid histological sections and labelled diagrams. In the same manner chapters on the body's tissue reaction and periodontal problems are covered.

Throughout the book emphasis is placed on an interdisciplinary approach to treatment with a chapter dedicated to explaining this concept and further independent chapters on the collaboration between orthodontics and periodontics, as well as the link with prosthetics. The book is instilled with evidence-based supported information throughout and explores contemporary techniques such as $3 \mathrm{D}$ visual treatment objective (VTO) for use in pre-treatment work-ups, and the use of mini implants for anchorage.

Current expectations of the adult patient places demands on the orthodontist to provide aesthetic and effective treatment in a shorter period of time, without the obvious appearance of an appliance. Choosing the correct, least complex appliance first time around can reduce treatment time. This book helps with the appliance selection, design and treatment sequencing. Advantages of the various aesthetic options are discussed, including the use of ceramic brackets, lingual and Invisalign ${ }^{\circledR}$ systems, alongside their limitations in comparison to the more conventional metal-based methods.

In summary, this is a comprehensive and advanced insight into adult orthodontics and one that would be clinically relevant for any specialist who is involved in the assessment and management of complex adult orthodontic cases.

M. Gormley 